\title{
Electrostatic confinement of electrons in an integrable graphene quantum dot
}

\author{
J. H. Bardarson, ${ }^{1}$ M. Titov ${ }^{2}$ and P. W. Brouwer ${ }^{1}$ \\ ${ }^{1}$ Laboratory of Atomic and Solid State Physics, Cornell University, Ithaca, NY 14853, USA \\ ${ }^{2}$ School of Engineering 89 Physical Sciences, Heriot-Watt University, Edinburgh EH14 4AS, UK
}

(Dated: November 10, 2018)

\begin{abstract}
We compare the conductance of an undoped graphene sheet with a small region subject to an electrostatic gate potential for the cases that the dynamics in the gated region is regular (discshaped region) and classically chaotic (stadium). For the disc, we find sharp resonances that narrow upon reducing the area fraction of the gated region. We relate this observation to the existence of confined electronic states. For the stadium, the conductance looses its dependence on the gate voltage upon reducing the area fraction of the gated region, which signals the lack of confinement of Dirac quasiparticles in a gated region with chaotic classical electron dynamics.
\end{abstract}

PACS numbers: 73.63.-b, 73.63.Kv, 73.23.-b

A characteristic feature of the massless Dirac electrons in graphene, a single layer of graphite, is the suppression of backscattering [1] and the resulting strong angle dependence of scattering at interfaces 2] (related to "Klein tunneling" [3, 4]). For clean graphene, graphene without atomic-scale defects or impurities that cause intervalley scattering, the transmission probability through an electrostatic barrier is unity at perpendicular incidence and drops sharply upon increasing the angle of incidence (cf. Fig. 1a). The absence of backscattering at perpendicular incidence is a consequence of a combination of an effective time reversal symmetry and band topology [1, 5$]$. The same combination is responsible for the fact that, generically, Dirac electrons can not be confined in electrostatically defined structures or by a smooth random electrostatic potential through Anderson localization [6, 7]. For this reason, most experimental [8, 9] and theoretical [10, 11] studies of confined electrons in graphene are performed on etched structures, in which inevitably intervalley scattering is strong and the physics essentially reduces to that of normal semiconductor quantum dots.

This general argument against the possibility to confine electrons electrostatically overlooks a special case: Dirac electrons can be confined electrostatically if their classical dynamics inside the gated area is integrable and the corresponding Dirac equation is separable. The integrable classical dynamics allows for the existence of electron paths for which the electron never approaches the boundary of the confinement area at perpendicular incidence. For such paths, an electrostatic barrier is fully reflecting, so that the electrons are effectively confined. In quantum mechanics, it is the corresponding separability of the Dirac equation that invalidates the topological arguments against the electrostatic confinement of electrons [6, 12].

The requirement of integrable dynamics is essential for the electrostatic confinement of the electrons. Certainly, if the classical dynamics is chaotic, electrons can not be confined electrostatically. There is a rich history of examples in which the question of integrability of the clas-

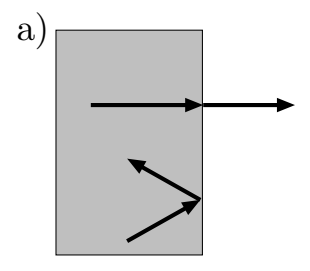

b)

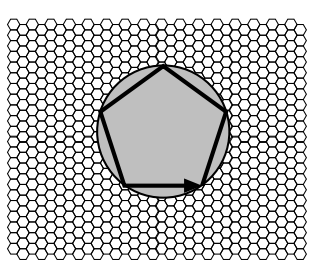

c)

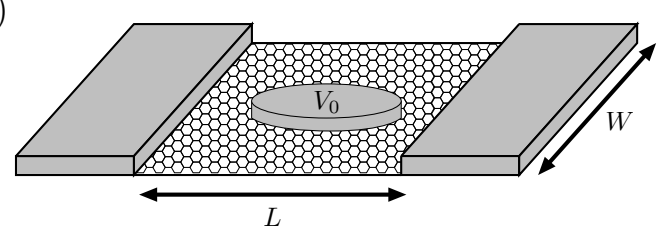

Figure 1: Angle-dependent scattering of an electrostatic interface (a), a circular quantum dot (defined by an electrostatic gate potential) in an infinite graphene sheet showing an example of a path that corresponds to a bound states (b), and a two terminal transport setup that can probe for the existence of bound states (c).

sical dynamics has profound consequences for a quantum mechanical system. The best known example is that of the level spacing distribution of ballistic normal-metal quantum dots 13, 14], which is described by WignerDyson statistics for quantum dots with chaotic classical dynamics, and Poisson statistics for quantum dots with integrable classical dynamics. Another example, similar in spirit to the present study, is the lifetime of resonant states in optical resonators or microlasers [15, 16]. Regular lasers have optical ray trajectories that always exceed the critical angle and thus have an infinite lifetime, while chaotic trajectories are always eventually refracted out and thus have a finite lifetime 17.

Experimentally, the existence of bound states would be clearly revealed in a two terminal conductance measurement setup schematically shown in Fig. 11k. A "buffer" of undoped graphene separates a gated area from the leads and acts as an effective barrier. (The barrier is only "ef- 
fective" because the conductivity of undoped graphene is $4 e^{2} / \pi h$, not zero.) A metal gate defines a "quantum dot" in which the carrier density is nonzero. If the dot is located approximately halfway between the metal contacts, eventual bound states in the dot will appear as resonances in the conductance upon varying the gate voltage of the dot. Since only regular dots can support bound states, the presence of sharp resonances in the two terminal conductance gives a clear experimental signature of integrability in the graphene quantum dot.

The remainder of this letter is reserved for material in support of the above scenario. We begin by considering, as in Fig. 1b, a circular quantum dot in an infinite graphene sheet. This problem can be solved exactly, and the location and character of the bound states can be identified explicitly. We then turn to the twoterminal transport geometry of Fig. 1: and compare numerical simulations of transport through a layer of undoped graphene with circular and stadium-shaped quantum dots

Taking the gate potential to be smooth on the scale of the lattice spacing, the electron dynamics in graphene is accurately described by the single valley Dirac Hamiltonian

$$
H_{0}=v_{\mathrm{F}} \mathbf{p} \cdot \sigma+V_{\text {gate }}(x, y)
$$

where $\sigma=\left(\sigma_{x}, \sigma_{y}\right)$ are Pauli matrices and $v_{\mathrm{F}}$ is the Fermi velocity. For a circular dot we take the gate potential to be

$$
V_{\text {gate }}(r)=\hbar v_{\mathrm{F}} V_{0} \vartheta(r-R),
$$

with $\vartheta$ the Heaviside step function. (The choice of a step function allows a closed-form solution; it is not essential for the existence of bound states. The structure of quasi-bound states in the inverted setup [zero potential inside, nonzero outside] was considered in Ref. 18.) We look for eigenstates of $H_{0}$ at zero energy. Because of the rotation symmetry of $V_{\text {gate }}$, the eigenstates can be labeled according to their angular momentum $m$, with $m$ half-integral [19]. They are

$$
\psi_{m}=e^{i m \theta}\left(\begin{array}{c}
e^{-i \theta / 2} \varphi_{m,+}(r) \\
e^{i \theta / 2} \varphi_{m,-}(r)
\end{array}\right),
$$

where the radial functions $\varphi_{m, \sigma}$ with $\sigma= \pm$ satisfy

$$
-i\left[\partial_{r}-\sigma\left(m-\frac{\sigma}{2}\right) \frac{1}{r}\right] \varphi_{m, \sigma}=-V_{0} \vartheta(R-r) \varphi_{m,-\sigma} .
$$

For $r>R$ the two equations (4) decouple and the solutions are

$$
\varphi_{m, \sigma}=a_{\sigma} r^{\sigma m-1 / 2} .
$$

Normalizability requires $|m| \geq 3 / 2$ and $a_{+}=0\left(a_{-}=0\right)$ for $m>0(m<0)$. For $r<R$ one finds

$$
\begin{aligned}
& \varphi_{m,+}(r)=b J_{|m-1 / 2|}\left(\left|V_{0}\right| r\right), \\
& \varphi_{m,-}(r)=-i b \operatorname{sgn}\left(V_{0} m\right) J_{|m+1 / 2|}\left(\left|V_{0}\right| r\right) .
\end{aligned}
$$

Continuity of the wave function at $r=R$ then gives the implicit equation

$$
J_{|m|-1 / 2}\left(\left|V_{0}\right| R\right)=0,
$$

from which one obtains the values of the gate voltage $V_{0}$ for which bound states exist at zero energy. These states correspond to states with nonzero orbital angular momentum that circulate inside the disc, thereby avoiding Klein tunneling. The states are twofold degenerate, corresponding to clockwise and anti-clockwise circulation. This twofold degeneracy is not specific to the circular shape of the quantum dot, but is rather an example of the Kramers degeneracy due to the effective time reversal, $\mathcal{T}=i \sigma_{y} \mathcal{C}$ with $\mathcal{C}$ complex conjugation, of the single valley Dirac Hamiltonian (11).

The bound states exist for $|m| \geq 3 / 2$ only. At angular momentum $|m|=1 / 2$ no normalizable bound state exists. Instead, the "zero mode" at $|m|=1 / 2$ is an extended state. It is the existence of this extended zero mode that is responsible for the fact that electrons can not be confined electrostatically in a chaotic dot: For a chaotic dot, all wavefunctions will in general have a finite contribution from the zero mode, which will determine the finite lifetime of the state. This is to be contrasted with the case of the circle studied above, which admits solutions to the Dirac equation in which the zero mode has vanishing weight.

Following Ref. 20, transport properties in the twoterminal geometry of Fig. 1r are described by the Hamiltonian

$$
H=H_{0}+U_{\text {lead }},
$$

where $U_{\text {lead }}(x)=0$ for $0<x<L$ and $U_{\text {lead }}(x) \rightarrow \infty$ otherwise. The graphene sheet has width $W$. The conductance is obtained numerically using the transfer matrix method of Refs. 21 and 7 . We place the center of the quantum dot at $x=L / 2$. In Fig. 2 we show the results of such a calculation for the conductance for an undoped graphene sheet with a circular quantum dot, as a function of the gate voltage $V_{0}$ for aspect ratios $W / L=1$ and $W / L=6$ and periodic boundary conditions in the transverse $(y)$ direction. In both cases, we observe sharp resonances on top of a background conductance from the finite conductivity of the undoped graphene buffer. The positions of the sharp resonances are in good agreement with the gate voltages (7) of the bound states. We have also performed the same calculation with antiperiodic boundary conditions (results not shown) and obtained results that are qualitatively similar to those for periodic boundary conditions if $W / L=1$ and indistinguishable from these if $W / L=6$ (as was to be expected [20]).

For aspect ratio $W / L=1$, the background conductance is essentially independent of the gate voltage $V_{0}$. For large aspect ratios, however, the background conductance has a relatively slow gate-voltage dependence 

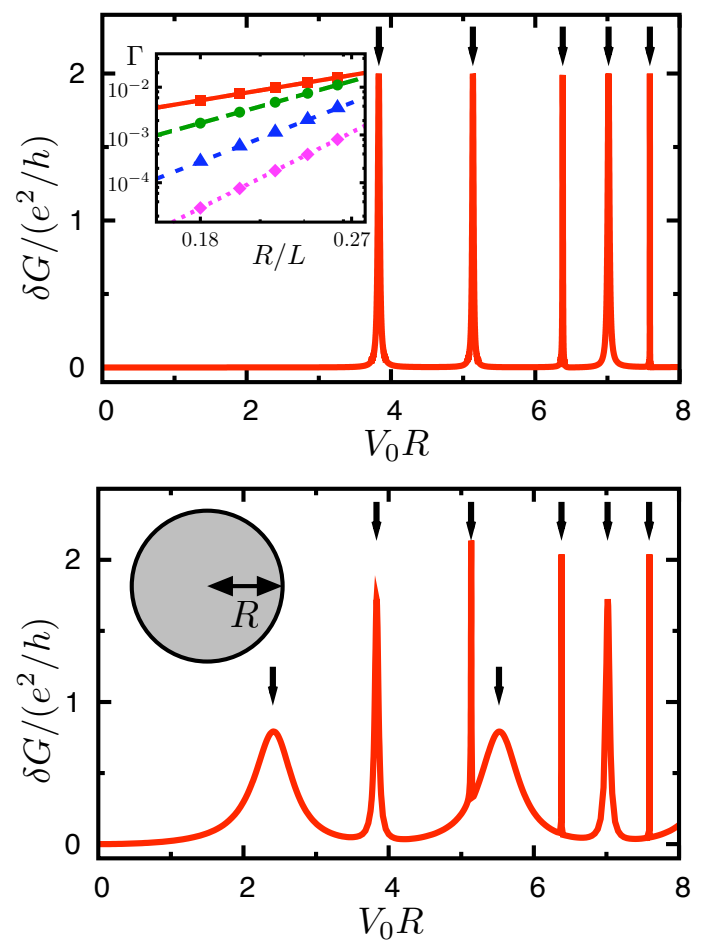

Figure 2: Conductance of an undoped graphene sheet with an electrostatically gated circular region of radius $R$ (lower inset) placed in the center of the sheet, as a function of the gate voltage $V_{0}$ for $R / L=0.26, W / L=1$ (upper panel) and $R / L=0.2, W / L=6$. The arrows show the positions of the solution to Eq. (7). Upper inset the dependence of the width of the first few resonances on dot size $R / L$.

from a contribution from the zero mode. This is a finite size effect. However, the density of states associated with the zero mode goes to zero only logarithmically in the limit $L / R \gg 1$, which is why this feature persists for the entire range of system sizes accessible numerically. Still, the sharp resonances from the true bound states can be unambiguously identified, and their positions are independent of aspect ratio and boundary conditions.

The width of the resonances in Fig. 2 depends on the angular momentum quantum number $m$ of the resonances. The dependence on $m$ and on the size $L$ of the undoped graphene buffer separating the dot from the electrodes can be understood in a simpler model that retains the circular symmetry. To that end we replace the leads by a circular electrode at a distance $L$ from the center of the quantum dot. Since the integrated probability of the bound states at distance $L$ from the origin scales as $(R / L)^{2 m}$, we conclude that the resonance width $\Gamma$ should have the same dependence on $L$ and $m$,

$$
\Gamma \sim(R / L)^{2|m|} .
$$

The larger the angular momentum quantum number $m$ the narrower the resonances become.
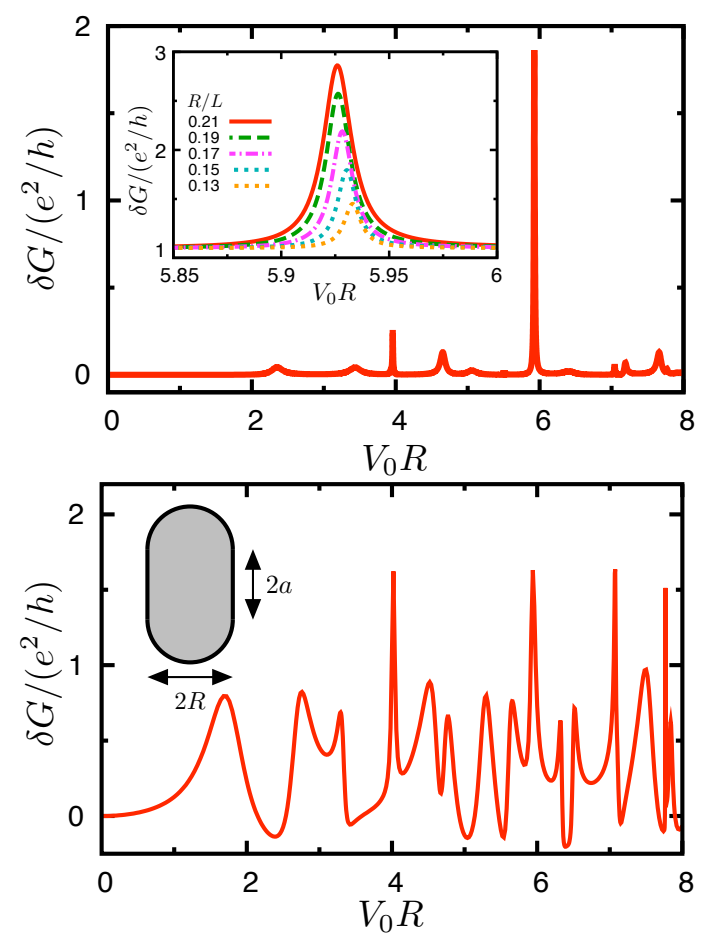

Figure 3: Same as Fig. 2 but with a stadium-shaped gated region (lower inset) with $2 a / R=\sqrt{3}$ and $R / L=0.21, W / L=$ 1 (upper panel) and $R / L=0.2, W / L=6$ (lower panel). The dependence of the sharpest resonance in the upper panel on the ratio $R / L$ is shown in the upper inset.

We have verified the prediction (9) for the resonance width by repeating the numerical calculation for different values of $R / L$. The resonances have a Lorentzian shape, and we extract their width by fitting to the formula

$$
\delta G=\frac{2 \Gamma^{2}}{\left(V_{0} R-x_{0}\right)^{2}+\Gamma^{2}} \frac{e^{2}}{h},
$$

where $x_{0}$ is approximately given by the zero of the Bessel function (7). (There is a small shift of the resonance position from the coupling to the leads.) The dependence of the resonance width on $R / L$ shown in the upper inset to Fig. 3 is in excellent agreement with the prediction (9) independent of boundary conditions and aspect ratio.

Other regular shapes that give rise to integrable classical dynamics and a separable Dirac equation behave in the same way as the circle. One analytically solvable example is given by a strip of width $R$ extending across the entire width of the sample. For $W \simeq L$ the conductance has separated Lorentzian resonances as a function of gate voltage, comparable to the circular gate voltage of Fig 2, reflecting discrete bound states. In the limit $R \ll L \ll W$ these bounds states become effectively degenerate and their resonances start to overlap. The resonances are at position $V_{0} R=\pi(n+1 / 2)$ with integer 


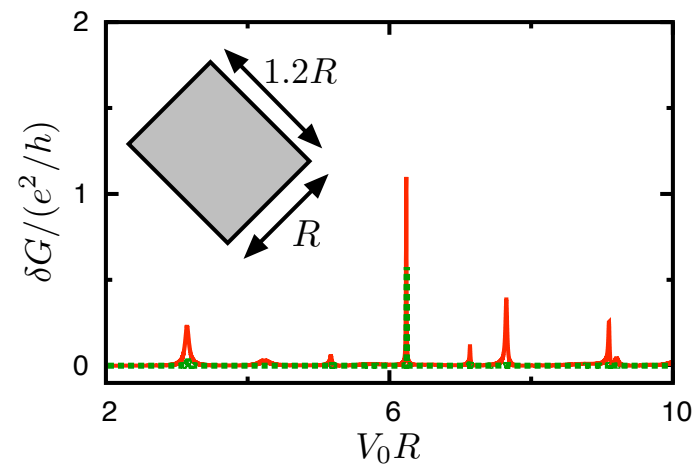

Figure 4: Same as Fig. 2, but with an an electrostatically gated rectangular region of size $R \times 1.2 R$ and rotated by 45 degrees relative to the leads placed in the center of the sheet. The solid and dotted curves correspond to $R / L=0.2$ and 0.1 , respectively.

$n$ and their height diverges (in the limit $U_{\text {lead }} \rightarrow \infty$ ),

$$
G=\frac{e^{2}}{h} \frac{W}{\pi L} \frac{1}{\sin V_{0} R} \ln \left|\frac{1+\sin V_{0} R}{\cos V_{0} R}\right| .
$$

As a prototypical example of a chaotic quantum dot we consider the conductance through a stadium (see lower inset in Fig. 3). We have given arguments for why the stadium can not support bound states and we thus do not expect to see any sharp resonances in the conductance. This is confirmed by the calculated conductance trace in Fig. 31, which shows wide features which have a height that decreases and a width that remains constant upon increasing $L / R$ (provided $L / R$ is large enough). This is shown explicitly in the inset to the upper panel of Fig. 3 for the sharpest conductance peak near $V_{0} R=6$. Just as in the circular case contributions from quasibound states appear for larger aspect ratios. The behavior of these features is very similar to the zero mode contributions in the circle.

For the Dirac equation, integrability of the classical equation of motion does not always imply integrability of the quantum mechanical equation of motion. An example is a uniformly gated region with a rectangular shape, for which the classical dynamics is integrable, but the Dirac equation is not [22]. For the gate voltage range we considered in our numerical calculations (up to $\sim 50$ levels in a circle of comparable size), we found no conductance resonances that have the same behavior upon increasing the ratio $L / R$ as resonances in a circular quantum dot (becoming narrower at constant height); in contrast all resonances in the range of gate potentials we considered become lower upon increasing $L / R$. (Typical data are shown in Fig. (4) This implies that, for this gate voltage range, a necessary condition for confinement is separability of the quantum mechanical equation of motion, not merely integrability of the classical dynamics.
We can not numerically determine whether sharp resonances and, hence, confined electronic states, emerge at higher gate voltages, where the quantum-classical correspondence plays a more significant role.

In conclusion, we have shown that gate potentials in which the (quantum) equation of motion is integrable, surrounded by undoped graphene, allow electrons to be confined. Gated regions in which the classical dynamics is chaotic, on the other hand, do not support bound states. Bound states manifest themselves in a two terminal conductance measurement through the presence of sharp resonances. Compared to the complexity of experiments on etched quantum dots in graphene, the setup suggested here is a promising candidate for the observation of signatures of integrable dynamics in graphene.

We are grateful to C. Kane for a stimulating discussion. This work was supported by the NSF under Grant No. DMR 0705476.

[1] T. Ando, T. Nakanishi, and R. Saito, J. Phys. Soc. Japan 67, 2857 (1998).

[2] V. V. Cheianov and V. I. Fal'ko, Phys. Rev. B 74, 041403(R) (2006).

[3] M. I. Katsnelson, K. S. Novoselov, and A. K. Geim, Nat. Phys. 2, 620 (2006).

[4] C. W. J. Beenakker, Rev. Mod. Phys. 80, 1337 (2008).

[5] S. Ryu, C. Mudry, H. Obuse, and A. Furusaki, Phys. Rev. Lett. 99, 116601 (2007).

[6] K. Nomura, M. Koshino, and S. Ryu, Phys. Rev. Lett. 99, 146806 (2007).

[7] J. H. Bardarson, J. Tworzydło, P. W. Brouwer, and C. W. J. Beenakker, Phys. Rev. Lett. 99, 106801 (2007).

[8] L. A. Ponomarenko, F. Schedin, M. I. Katsnelson, R. Yang, E. W. Hill, K. S. Novoselov, and A. K. Geim, Science 320, 356 (2008).

[9] C. Stampfer, E. Schurtenberger, F. Molitor, J. Güttinger, T. Ihn, and K. Ensslin, Nano Lett. 8, 2378 (2008).

[10] J. Wurm, A. Rycerz, I. Adagideli, M. Wimmer, K. Richter, and H. U. Baranger, Phys. Rev. Lett. 102, 056806 (2009).

[11] F. Libisch, C. Stampfer, and J. Burgdörfer, Phys. Rev. B 79, 115423 (2009).

[12] L. Fu and C. L. Kane, Phys. Rev. B 74, 195312 (2006).

[13] C. W. J. Beenakker, Rev. Mod. Phys. 69, 731 (1997).

[14] Y. Alhassid, Rev. Mod. Phys. 72, 895 (2000).

[15] Y. Yamamoto and R. E. Slusher, Phys. Today 46, No. 6, 66 (1993).

[16] B. R. Johnson, J. Opt. Soc. Am. A 10, 343 (1993).

[17] J. U. Nöckel and A. D. Stone, Nature 385, 45 (1997).

[18] A. Matulis and F. M. Peeters, Phys. Rev. B 77, 115423 (2008); P. Hewageegana and V. Apalkov, Phys. Rev. B 77, 245426 (2008).

[19] P. Recher, B. Trauzettel, A. Rycerz, Y. M. Blanter, C. W. J. Beenakker, and A. F. Morpurgo, Phys. Rev. B 76, 235404 (2007).

[20] J. Tworzydło, B. Trauzettel, M. Titov, A. Rycerz, and C. W. J. Beenakker, Phys. Rev. Lett. 96, 246802 (2006).

[21] M. Titov, Europhys. Lett. 79, 17004 (2007).

[22] M. V. Berry and R. J. Mondragon, Proc. R. Soc. A 412, 53 (1987). 